\title{
Colegio Médico de Honduras: Reconocimiento Médico del Año
}

\author{
Colegio Médico de Honduras: Physician of the Year Award
}

\author{
Ana Ligia Chinchilla, ${ }^{1}$ Rosa María Duarte. ${ }^{2}$
}

${ }^{1}$ Médico Especialista en Ginecología y Obstetricia; Facultad de Ciencias Médicas, Universidad Nacional Autónoma de Honduras; Instituto Hondureño de Seguridad Social. ${ }^{2}$ Médico Salubrista; Dirección General de Normalización, Secretaría de Salud de Honduras; Tegucigalpa.

\section{INTRODUCCIÓN}

El Colegio Médico de Honduras $(\mathrm{CMH})$ a través de sus diferentes Secretarías ha identificado diversas actividades para apoyar, reconocer y promover el trabajo gremial y aportes al campo de la medicina y a la sociedad. Una de estas actividades la ha constituido el otorgamiento de reconocimientos y premios. Anualmente el $\mathrm{CMH}$ otorga el reconocimiento Médico del Año.

\section{RECONOCIMIENTO MÉDICO DEL AÑO}

El reconocimiento Médico del Año otorgado por el $\mathrm{CMH}$, es un reconocimiento público al médico que se ha destacado por su actividad profesional de máxima calidad. Este reconocimiento se otorga en los ámbitos de medicina hospitalaria, atención primaria, salud pública, mental, atención socio sanitaria, investigación biomédica y humanidades médicas. Su objetivo es reconocer la obra de aquellos médicos que, con apego estricto a la ética médica, hayan sobresalido entre sus pares y sean un modelo a seguir en lo humano y profesional por su trayectoria de excelencia, creatividad, aporte relevante al desarrollo y prestigio de la medicina nacional. ${ }^{1}$ El reconocimiento surgió como una iniciativa de la Junta Directiva del $\mathrm{CMH}$ en el año 2002, estando ésta constituida por Dra. Odessa Henríquez, Presidenta; Dr. Reynaldo Gómez Urtecho, Vice-Presidente; Dra. Julia Medal Mendoza, Secretaria de Actas y Correspondencia; Dr. Rafael Francisco Aguilar, Secretario de Finanzas; Dra. Rosa Edith Alvarenga, Secretaria de Colegiaciones; Dr. Elmer López Lutz, Secretario de Asuntos Educativos y Culturales; Dra. Rutilia Calderón, Secretaria de Acción Social; Dr. José Manuel Espinal, Fiscal; y Dr. Francisco Herrera Alvarado, Vocal I. ${ }^{2}$

Para otorgar este reconocimiento el médico debe de reunir los siguientes requisitos: ser hondureño, médico graduado, legalmente inscrito en el CMH y en pleno goce de sus derechos, ser de reconocida honorabilidad, de intachable moral y ética profesional, con alto espíritu laboral y estar en el ejercicio

Recibido: 04-03-2020; Aceptado para publicación 27-07-2020

Dirección para correspondencia: Dra. Rosa María Duarte

Correo electrónico: duarte2000sl@yahoo.com

Declaración de relaciones y actividades financieras y no financieras y conflictos de interés: ninguna.

DOI: https://doi.org/10.5377/rmh.v88iSupl.1.11675 de sus funciones ya sea por acción directa de su profesión en atención en el cuidado de la salud de los hondureños a través de la investigación científica, así como otros méritos comprobados en su trayectoria, dar fe comprobada de su obra social con relevante acción humanitaria y/o científica en pro de los pacientes, con el gremio médico y la población en general. Asimismo, se describe que cualquier otro mérito no incluido en la descripción anterior será objeto de estudio de comprobación por la Comisión Médica Ad-Hoc para la valoración correspondiente. ${ }^{3}$ En los inicios del reconocimiento se contaba con el patrocinio de una institución farmacéutica por lo que al médico galardonado se le entregaba una placa de reconocimiento y un reconocimiento monetario; posteriormente se decidió la omisión del patrocinio externo. Actualmente se otorga la placa de reconocimiento entregada en un acto especial celebrado durante la Semana del Médico, promocionando el evento y difundiéndose a nivel nacional a través de las redes sociales del $\mathrm{CMH}$ (Comunicación personal, Dra. Odessa Henríquez, Dr. Elmer López Lutz, Junta Directiva 2002-2004, Tegucigalpa).

Para el proceso de selección del Médico del Año, los requisitos anteriormente descritos se hacen llegar por parte de la Junta Directiva a las Delegaciones y Sub-Delegaciones Médicas. Estas envían sus candidatos y después del cierre de la recepción de candidaturas, inicia un proceso de valoración llevada a cabo por la Comisión Médica Ad-Hoc conformada para tal efecto. Una vez seleccionado el médico galardonado, la selección es presentada para aprobación por la Junta Directiva. Cuando se completa el proceso de selección, al médico galardonado se le envía una nota comunicándole que por sus méritos ha sido galardonado con el reconocimiento y se le invita a la ceremonia especial para hacer entrega del mismo. Los reconocimientos han sido otorgados anualmente desde el año 2002, a excepción del período 2011-2015 en el cual no se realizó el proceso de selección. A la fecha los médicos galardonados representan una gama amplia de especialidades y sub-especialidades médicas, demostrando méritos indiscutibles y una gran capacidad profesional y de servicio (ver Cuadro 1). ${ }^{4}$ 
Cuadro 1. Lista de los médicos galardonados con el Reconocimiento Médico del Año, Colegio Médico de Honduras, 2002 - 2019.

\begin{tabular}{lll}
\hline Año & Médico Galardonado & Especialidad \\
\hline 2002 & Dr. Thirzo Bendaña & Oftalmología \\
2003 & Dr. Asdrubal Raudales & Psiquiatría \\
2004 & Dr. Pedro O. Fiallos & Cardiología \\
2005 & Dra. Flora Duarte & Oncología Clínica \\
2006 & Dr. Carlos Lagos & Cirugía \\
2007 & Dr. Edgardo Navarrete & Oftalmología \\
2008 & Dr. Mario Leon Goméz & Psiquiatría \\
2009 & Dr. Mario Rivera Williams & Pediatría; Maestría Salud Pública \\
2010 & Dr. Carlos R. García & Urología \\
$2016 *$ & Dr. José A. Amador Uclés & Psiquiatría \\
2017 & Dr. Ricardo L. Domínguez C. & Medicina Interna \\
2018 & Dr. Juan Almendarez Bonilla & Fisiología (MSc, Doctor Honoris \\
& & Causa) \\
2019 & Dra. Jackeline Alger & Parasitología (MSc, PhD) \\
\hline
\end{tabular}

Fuente: Archivos, Secretaria de Actas y Correspondencia, Colegio Médico de Honduras (Ref. 4). *En el período 2011 a 2015 el reconocimiento Médico del Año no fue otorgado.

\section{CONCLUSIÓN}

Consideramos que este reconocimiento es muy importante porque permite destacar la labor que el médico realiza en silencio diariamente en beneficio de los pacientes, incluyendo actividades en investigaciones que permiten el avance científico y su aporte al desarrollo de la humanidad, impulsado y respal- dado por los conceptos expresados en el juramento médico. ${ }^{5}$ Este reconocimiento es un estímulo para las nuevas generaciones y sus formadores, para siempre procurar la excelencia en el ejercicio profesional y una vida regida por principios éticos y guiada por los más altos estándares de profesionalismo, constituyéndose en una vocación y no en una simple ocupación..$^{6,7} \mathrm{El}$ $\mathrm{CMH}$ ha contado con otros reconocimientos y premios a la labor del médico incluyendo la educación médica continua. ${ }^{8,9}$

En vista que la comunidad médica se expande en las diferentes regiones de Honduras, siendo los médicos líderes en sus respectivas regiones y comunidades, se recomienda que el reconocimiento pudiera ser extensivo a cada una de las Delegaciones Médicas con el propósito de destacar la labor regional de nuestros colegas y que los candidatos sean seleccionados de forma equitativa tanto hombres como mujeres. También se recomienda la elaboración de un reglamento de tal manera que este pueda ser aprobado en la Asamblea General del Colegio Médico de Honduras y recolectar los aportes de los agremiados. El CMH se prestigia al honrar a colegas con tan altos méritos.

\section{CONTRIBUCIONES}

Ambas autoras identificaron el tema y lo desarrollaron de manera conjunta. RMD identificó los documentos gremiales. Ambas autoras atendieron las recomendaciones editoriales y aprobaron la versión final del artículo.

\section{AGRADECIMIENTO}

Se agradece a la Dra. Odessa Henríquez, Facultad de Ciencias Médicas, UNITEC, y al Dr. Elmer López Lutz, Departamento de Medicina Interna, Hospital Escuela, Tegucigalpa, por la información brindada.

\section{REFERENCIAS}

1. Colegio Médico de Honduras. Secretaría de Actas y Correspondencia. Punto de Acta 1808, año 2002, asuntos del Centro Nacional de Educación Médica Continua. Colegio Médico de Honduras. Tegucigalpa. Colegio Médico de Honduras; 2002.

2. Colegio Médico de Honduras. Secretaría de Actas y Correspondencia. Asamblea General Ordinaria. Acta, Número XLI (41). Tegucigalpa: Colegio Médico de Honduras; 2002.

3. Colegio Médico de Honduras. Secretaría de Asuntos Educativos y Culturales. Hoja de requisitos para optar a médico del año. Tegucigalpa: Colegio Médico de Honduras; 2002.

4. Colegio Médico de Honduras. Secretaría de Actas y Correspondencia. Archivos Período 2002-2019. Tegucigalpa: Colegio Médico de Honduras; 2002-2019.
5. Bueso Pineda A. El juramento médico. Rev Méd Hondur .2004; 72: 114115.

6. Pineda EB, Varela MC. Premio de Educación Médica Continua Jorge Haddad Quiñónez. Rev Méd Hondur. 2004; 72: 116-117.

7. Bu Figueroa E, Alger J, López Lutz E. Dr. Carlos Javier A. Zepeda: Premio de Educación Médica Continua Dr. Jorge Haddad Quiñónez, año 2003. Rev Méd Hondur. 2004; 72: 160-162.

8. Hernández-Escobar C, Hernández-Camarena J, González-González L, Barquet-Muñoz S. Formación en ética y profesionalismo para las nuevas generaciones de médicos. Persona y Bioética. 2010;14(1):30-39.

9. Romero Leguizamón CR. ¿Medicina: arte o ciencia? Una reflexión sobre las artes en la educación médica. Educación Médica. 2018; 19 (6): 359368. 